\section{Karin Maria Graos Salazar ${ }^{1}$} Miguel Perea $\mathrm{Paz}^{2}$

\section{${ }^{1}$ Ciruajno - Dentista}

2Docente del Departamento Académico de Estomatología del Niño y el Adolescente. Facultad de Estomatología. Universidad Peruana Cayetano Heredia.

\section{Correspondencia}

Miguel Perea Paz

Av. Honorio Delgado 430 Lima - 31, Perú

Av. Honorio Delgado 430 Lima

e-mail: miguelpp@upch.edu.pe

\title{
Medicación analgésica postquirúr- gica en pacientes atendidos en el Servicio de Odontología Pediátrica de la Clínica Estomatológica Cen- tral Cayetano Heredia (2000-2004)
}

Graos-Salazar K, Perea-Paz M. Medicación analgésica postquirúrgica en pacientes atendidos en el Servicio de Odontología Pediátrica de la Clínica Estomatológica Central Cayetano Heredia (2000-2004). Rev Estomatol Herediana. 2007; 17(2):53-57.

\section{RESUMEN}

El presente estudio tuvo como propósito determinar la frecuencia en el uso de medicamentos analgésicos posquirúrgicos prescritos en pacientes atendidos en un Servicio de Odontología Pediátrica. El grupo estuvo constituido por 91 historias clínicas de pacientes pediátricos sometidos a intervenciones quirúrgicas menores en el periodo del 2000 al 2004. La distribución de la muestra según sexo fue: $37,4 \%$ mujeres y $62,6 \%$ varones siendo el promedio de edad 8,82+2,43 años. En el análisis de los resultados se utilizo la distribución de frecuencia para determinar los tipos de cirugía, tipo y número de fármacos. Los resultados revelan que la exodoncia de dientes supernumerarios fue la cirugía más frecuente en ambos sexos $(47,4 \%)$. En relación a los tipos de cirugía oral según edad la exodoncia de diente supernumerario fue más frecuente en niños de 7 a 11 años (53,1\%); mientras que la frenectomia lingual fue la más frecuente en niños de 4 a 6 años. De las 91 HC solo 57 registraron medicación analgésica posquirúrgica. El número promedio de analgésicos recetados fue $2,11+0,86$. Según covariables se encontró que se recetó un mayor número de analgésicos en los varones $(2,18+0,92)$, en los niños de 7 a 11 años de edad $(2,36+0,82)$; y cuando se realizó exodoncia de diente supernumerario $(2,56+0,64)$. El Ketorolaco fue el analgésico mas recetado en varones $(34,9 \%)$, en los niños de 7 a 11 años $(38,4 \%)$ y en exodoncia de dientes retenidos $(42,1 \%)$. El Paracetamol fue el analgésico más recetado en mujeres (35,1\%), en los demás grupos de edad (75\%, 80\% y 50\% en niños de 1 a 3, 4 a 6 y 12 años a mas) y en frenectomia lingual (83,3\%). Finalmente se encontró que el ketorolaco y el naproxeno sódico fueron más frecuentemente recetados en exodoncia de diente supernumerarios (34,8\% en ambos casos).

Palabras clave: ANALGÉSICOS / CIRUGÍA BUCAL / ODONTOLOGÍA PEDIÁTRICA.

Post surgical Pain medication in patients treated at the Pediatric Dentistry Clinic of the Cayetano Heredia Dental Clinic (2000-2004)

ABSTRACT

The purpose of this study was to determine the frequency of usage of post surgery pain medication in patients treated at the Pediatric Dental Clinic. The group consisted of 91 charts from pediatric patients who received minor surgical procedures in the period between 2000 and 2004. The sample distribution was: $37.4 \%$ girls, $62.6 \%$ boys, with an average of $8.82+2.43$ years old. The analysis of the results used the distribution of frequencies to determine the type of surgeries performed and the type and number of medication. The results revealed that extraction of supernumerary teeth was the most frequent surgery for both sexes (47.4\%). In relation to the type of oral surgery by age, extraction of supernumerary teeth was the most frequent in 7 to11 year old children (53.1\%), while lingual frenectomy was the most frequent in 4-6 year old children. The average number of prescribed analgesics was $2.11+0.86$. By covariables it was found that more analgesics were prescribed in boys $(2.18+0.92)$, in 7 to 11 year old children $(2.36+0.82)$; and when the extraction of a supernumerary tooth was done $(2.56+0.64)$. Ketorolac was the most frequent analgesic prescribed in boys (34.9\%), in 7-11 year old children $(38.4 \%)$ and for extractions of retained teeth $(42.1 \%)$. Paracetamol was the most common analgesic prescribed in girls $(35.1 \%)$, in the other age groups $(75 \%, 80 \%$ and $50 \%$ en children aged 1 to 3, 4 to 6 and 6 to 12, respectively) and in lingual frenectomies (83.3\%). Finally it was found that ketorolac and naproxen were the most frequently prescribed after extraction of supernumerary teeth (34.8\% in both cases).

Key words: ANALGESICS / ORAL SURGERY / PEDIATRIC DENTISTRY.

\section{Introducción}

La Sociedad Internacional para el Estudio del Dolor define el dolor como una "Experiencia sensorial o emocional no placentera, producida por un daño tisular actual o potencial, o descrita en términos de esta" (1).

El dolor posoperatorio puede ocurrir en muchos contextos, sumándo- se al dolor incisional, asociado al dolor somático y visceral secundario (2).

En cuanto a los efectos adversos fisiológicos, el dolor es la respuesta al estrés que afecta el metabolismo y funcionamiento de los diferentes órganos $(2,3)$.

En lo referente a los efectos adversos psicológicos, el dolor induce a la incomodidad y a la ansiedad. Cuando la ansiedad es aguda, se puede elevar la percepción del dolor; cuando la ansiedad es prolonga$\mathrm{da}$, se puede experimentar angustia y resentimiento (2-4).

Los cambios fisiológicos dinámicos ocurridos durante el crecimiento y desarrollo exigen prestar atención particular al tratamiento 
farmacológico en niños. Cuando se atiende a niños, algunas de las diferencias principales en la farmacocinética ocurren en las áreas de: asimilación, absorción, distribución, sensibilidad del sitio receptor, metabolismo, y excreción. Aparte existen otros cambios de tipo anatómico y fisiológico que afectan el tratamiento en infantes (5).

Las dosis pediátricas generalmente se calculan con unos márgenes terapéuticos, con dosis máximas y mínimas que pueden utilizarse. Para evitar consecuencias funestas para los niños y los médicos, es imprescindible conocer las dosis de los medicamentos recomendadas para el grupo pediátrico. Desgraciadamente, el tratamiento farmacológico con frecuencia fracasa porque la dosis es demasiado baja o produce efectos adversos porque es demasiado alta (6).

Los analgésicos se clasifican en narcóticos y no narcóticos, dentro de los no narcóticos se encuentran los AntiInflamatorios No ESteroideos (AINES); estos son indicados en intervenciones quirúrgicas menores.

Los AINES que son utilizados hoy en día, en su inmensa mayoría inhiben la ciclooxigenasa 1 (COX1) presente en diversos tejidos $y$ median a las reacciones fisiológicas y a la ciclooxigenasa 2 (COX-2) presente en el tejido lesionado $(7,8)$.

Los AINES incluyen muy diversos compuestos, que aunque casi nunca tiene relación química alguna, si comparten actividades terapéuticas y efectos colaterales. En este grupo se incluyen los fármacos antinflamatorios, analgésicos antipiréticos y antiagregante plaquetario $(7,8)$.

Consideraciones para prescribir AINES.

- Los AINES son equipotentes, pero puede haber diferencias en la res- puesta individual.

- Su tolerancia es individual, pero puede variar aun entre preparaciones del mismo fármaco.

- Si un AINES no es efectivo se debe probar con otro hasta encontrar la respuesta deseada.

- Nunca se deben usar dos o más AINES al mismo tiempo, ya que el potencial tóxico se multiplica.

- Los estudios han demostrado que los AINES pueden ser requeridos para manejar algún nivel de dolor posoperatorio.

- Los AINES pueden ser analgésicos más efectivos si ellos son dados muy tempranamente y a dosis suficientes para prevenir la síntesis de prostaglandinas. Por lo tanto, se deberá considerar una dosis inicial de carga, tal como la dosis de mantenimiento, ambas condiciones permitiría alcanzar niveles terapéuticos más rápidamente.

- Prescribir AINES los dos primeros días después del procedimiento.

- El analgésico puede entonces ser tomado después del periodo inicial.

- Existen numerosos efectos adversos con los AINES pero sin embargo son mejor tolerados en pacientes saludables que los opiodes.

- Prescribir AINES mejor conocidos por el odontopediatra y que estén ala alcance del paciente.

- El paciente alérgico a un AINE puede ser alérgico a todos.

- Al seleccionar un AINE se debe considerar su seguridad, eficacia, tolerancia, costo conveniencia por dosis, presentación, vías y horarios.

- Cuando se administra un AINE conjuntamente con un analgésico narcótico es posible utilizar dosis menores de este último, siendo posible un mejor control del dolor con menores efectos indeseables.

- Se debe hacer una selección cui- dados de pacientes candidato a AINES. Dentro del grupo de mayor riesgo se encuentran los niños (22).

El objetivo del presente estudio fue determinar en que medida se prescribieron medicamentos analgésicos postquirúrgicos en pacientes atendidos en el Servicio de odontología pediátrica de la Clínica Estomatológica Central Cayetano Heredia.

\section{Material y métodos}

Se seleccionaron 91 historias clínicas de pacientes sometidos a intervenciones quirúrgicas menores, atendidos en el Servicio de Odontología Pediátrica de la Clínica Estomatológica Central en el periodo 2000-2004.

Los criterios de selección fueron: pacientes tratados por un solo operador, sin compromiso sistémico, atendidos con anestesia local y sin prescripción preoperatoria.

Los tipos de cirugía menor efectuadas se registraron como: frenectomia lingual, exodoncia de diente retenido, exodoncia de diente supernumerario, enucleación de odontoma, operculectomía, eliminación de mucocele.

Se registró el tipo de analgésico recetado luego de realizado la intervención quirúrgica.

\section{Resultados}

El presente estudio se realizó en base a la revisión de 91 historias clínicas (HC) del Área de Odontología Pediátrica de la Clínica Estomatológica Central de la Universidad Peruana Cayetano Heredia, correspondientes a pacientes que fueron sometidos a cirugía oral menor entre los años 2000 y 2004. De las 91 historias clínicas revisadas, el $62,6 \%$ correspondió varones, siendo la edad promedio de $8,82+2,43$ años. 
En relación con los tipos de cirugía oral menor según sexo (Tabla 1), la exodoncia de dientes supernumerarios fue la más frecuentemente en varones (47,4\%).

En relación con los tipos de cirugía oral menor según edad (Tabla 2) se encontró que la exodoncia de dientes supernumerario fue la cirugía más frecuente en niños de 7 a 11 años (53,1\%).

Sólo 57 HC registraron la medicación analgésica post-quirúrgica, con un total de 120 medicamentos recetados. El número promedio de analgésicos posquirúrgicos recetados fue de 2,11+0,86.

En relación al número de analgésicos posquirúrgicos recetados se encontró que se recetó un mayor número de analgésicos en el sexo masculino $(2,18+0,92)$, en los niños de 7 a 11 años de edad $(2,36+0,82)$, y cuando se realizó exodoncia de dientes supernumerarios $(2,56+0,64)$.

En relación con el tipo de analgésicos posquirúrgicos recetados según sexo (Tabla 3), se encontró que el Ketorolaco fue el analgésico más recetado en el sexo masculino $(34,9 \%)$ mientras que el Paracetamol lo fue en el sexo femenino (35,1\%).

Con respecto a los analgésicos recetados luego de las intervenciones quirúrgicas según edad (Tabla 4), se observó que el Ketorolaco fue el analgésico más recetado en los niños de 7 a 11 años (38,4\%), mientras que el Paracetamol lo fue en los demás grupos de edad (75\%, $80 \%$ y $50 \%$ en los niños de 1 a 3, 4 a 6 y 12 años a más).

En relación con el tipo de analgésicos posquirúrgicos recetados según cirugía oral menor (Tabla 5), se encontró que el Paracetamol fue el analgésico más recetado en frenectomía lingual (83,3\%), mien-

Tabla 1. Tipos de cirugía oral menor en pacientes atendidos en el servicio de odontología pediátrica según sexo (2000-2004).

\begin{tabular}{lccrr}
\hline Tipos de cirugía & $\begin{array}{l}\text { Masculino } \\
\mathrm{n}(\%) \\
(\%)\end{array}$ & $\begin{array}{c}\text { Femenino } \\
\mathrm{n}\end{array}$ & \multicolumn{2}{c}{ Total } \\
$\mathrm{n}$ & & & \\
\hline Frenectomía lingual & $5(8,8)$ & $4(11,8)$ & 9 & $(9,9)$ \\
$\begin{array}{l}\text { Exodoncia de diente } \\
\text { retenido }\end{array}$ & $8(14,0)$ & $6(17,6)$ & 14 & $(15,4)$ \\
Exodoncia de diente & & & & \\
supernumerario & $27(47,4)$ & $16(47,1)$ & 43 & $(47,3)$ \\
Enucleación de odontoma & $4(7,0)$ & $2(5,9)$ & 6 & $(6,6)$ \\
Operculectomía & $11(19,3)$ & $5(14,7)$ & 16 & $(17,6)$ \\
Eliminación de mucocele & $2(3,5)$ & $1(2,9)$ & 3 & $(3,3)$ \\
Total & 57 & 34 & 91 & \\
\hline
\end{tabular}

Tabla 2. Tipos de cirugía oral menor practicada en pacientes atendidos en el servicio de odontología pediátrica según edad (2000-2004).

\begin{tabular}{|c|c|c|c|c|c|}
\hline $\begin{array}{l}\text { Grupo etario } \\
\text { Tipos de cirugía }\end{array}$ & $\begin{array}{l}1 \text { a } 3 \\
\text { n (\%) }\end{array}$ & $\begin{array}{l}4 \text { a } 6 \\
\text { n }(\%)\end{array}$ & $\begin{array}{l}7 \text { a } 11 \\
\text { n }(\%)\end{array}$ & $\begin{array}{l}12 \text { a más } \\
\text { n (\%) }\end{array}$ & $\begin{array}{l}\text { Total } \\
\mathrm{N}(\%)\end{array}$ \\
\hline Frenectomía lingual & $1(33,3)$ & $4(40,0)$ & $4(6,3)$ & 0 & $9(9,9)$ \\
\hline $\begin{array}{l}\text { Exodoncia de diente } \\
\text { retenido }\end{array}$ & 0 & 0 & $12(18,8)$ & $2(14,3)$ & $14(15,4)$ \\
\hline $\begin{array}{l}\text { Exodoncia de diente } \\
\text { supernumerario }\end{array}$ & 0 & $3(30$ & $34(5$ & $6(42$ & $43(47,3)$ \\
\hline de odontoma & $1(33,3)$ & 0 & $4(6,3)$ & $1(7,1)$ & $6(6,6)$ \\
\hline Operculectomía & 0 & $2(20,0)$ & $10(15,6)$ & $4(28,6)$ & $16(17,6)$ \\
\hline Eliminación de mucocele & $1(33,3)$ & $1(10,0)$ & 0 & $1(7,1)$ & $3(3,3)$ \\
\hline Total & 3 & 10 & 64 & 14 & 91 \\
\hline
\end{tabular}

Tabla 3. Tipos de analgésicos posquirúrgicos recetados a pacientes atendidos en el servicio de odontología pediátrica según sexo (2000-2004).

\begin{tabular}{lccc}
\hline Tipos de analgésico & \multicolumn{2}{c}{ masculino } & \multicolumn{2}{c}{ femenino } & total \\
\hline Ketorolaco & $\mathrm{n}(\%)$ & $\mathrm{n}(\%)$ & $\mathrm{n}(\%)$ \\
Paracetamol & $29(34,9)$ & $9(24,3)$ & $38(31,7)$ \\
Naproxeno & $27(32,5)$ & $13(35,1)$ & $40(33,3)$ \\
Ibuprofeno & $24(28,9)$ & $12(32,4)$ & $36(30,0)$ \\
Diclofenaco+Paracetamol & 0 & $1(2,7)$ & $1(0,8)$ \\
Rofecoxib & $1(1,2)$ & $1(2,7)$ & $2(1,7)$ \\
Total & $2(2,4)$ & $1(2,7)$ & $3(2,5)$ \\
\hline
\end{tabular}

Tabla 4. Tipos de analgésicos posquirúrgicos recetados a pacientes atendidos en el servicio de odontología pediátrica según edad (2000-2004).

\begin{tabular}{llllll}
\hline Grupo etario & $\begin{array}{l}\text { 1 a } 3 \\
\text { Tipos de analgésico }\end{array}$ & $\begin{array}{l}4 \text { a } 6 \\
\mathrm{n}(\%)\end{array}$ & $\mathrm{n}(\%)$ & $\mathrm{n}$ a 11 & \multicolumn{2}{l}{12 a más } & \multicolumn{2}{c}{ Total } \\
$\mathrm{n}(\%)$ & $\mathrm{N}(\%)$ \\
\hline Ketorolaco & 0 & 0 & $38(38,4)$ & 0 & $38(31,7)$ \\
Paracetamol & $3(75,0)$ & $4(80,0)$ & $27(27,3)$ & $6(50,0)$ & $40(33,3)$ \\
Naproxemo & $1(25,0)$ & 0 & $30(30,3)$ & $5(41,7)$ & $36(30,0)$ \\
Ibuprofeno & 0 & $1(20,0)$ & 0 & 0 & $1(0,8)$ \\
Diclofenaco+Paracetamol & 0 & 0 & $2(2,0)$ & 0 & $2(1,7)$ \\
Rofecoxib & 0 & 0 & $2(2,0)$ & $1(8,3)$ & $3(2,5)$ \\
Total & 4 & 5 & 99 & 12 & 120 \\
\hline
\end{tabular}

tras el Ketorolaco lo fue en exodoncia de dientes retenidos
(42,1\%) y enucleación de odontoma (42,9\%). Finalmente, el Ketorolaco 
y el Naproxeno sódico fueron más frecuentemente recetados en exodoncia de dientes supernumerarios (34,8\% en ambos casos).

\section{Discusión}

En múltiples estudios se revela que el paracetamol es el analgésico de elección para el manejo del dolor postoperatorio, tanto en intervenciones orales como en otro tipo de cirugías ambulatorias (circuncisión, tonsilectomías) $(9,10)$.

Jurgens et al. (11) realizaron un estudio en 72 pacientes sometidos a extracciones dentales bajo anestesia general, en los cuales evaluaron la eficacia de analgesia del fentanilo y el paracetamol a las 24 horas; concluyendo que la mayoría de pacientes estaban libres del dolor independientemente del analgésico utilizado.

Pedreira et al. (12) en 1999 encuentran en 297 niños entre 5 y 12 años sometidos a extracción dental, que el ibuprofeno fue superior al paracetamol en el alivio del dolor posoperatorio.

A pesar que la literatura confiere mayor superioridad en el manejo del dolor posexodoncia en niños al ibuprofeno frente al paracetamol, en nuestro estudio fue el analgésico menos recetado debido probablemente a los efectos adversos del ibuprofeno con respecto al paracetamol.

Haas (13) considera para el manejo del dolor dental como analgésicos ideales al Paracetamol e Ibuprofeno. Además señala que el dolor posoperatorio crónico se resuelve mejor con AINES teniendo especial consideración de los malestares gástricos que muchas veces estos producen.

Mehlisch et al. (14) determinaron que el Ibuprofeno nuestra una mejor analgesia en el manejo del dolor posoperatorio moderado a se-

Tabla 5. Tipos de analgésicos posquirúrgicos recetados a pacientes atendidos en el servicio de odontología pediátrica según cirugía oral menor (2000-2004)

\begin{tabular}{|c|c|c|c|c|c|c|}
\hline \multirow{2}{*}{$\begin{array}{l}\text { cirugía } \\
\text { analgésico }\end{array}$} & FL & ER & ES & OD & $\mathrm{OP}$ & \multirow{2}{*}{$\begin{array}{l}\text { EM } \\
n(\%)\end{array}$} \\
\hline & (\%) & n $\quad(\%)$ & $(\%)$ & $(\%)$ & n $\quad(\%)$ & \\
\hline Ketorolaco & 0 & $8(42,1)$ & $24(34,8)$ & $6(42,9)$ & 0 & 0 \\
\hline Paracetamol & $5(83,3)$ & $6(31,6)$ & $19(27,5)$ & $3(21,4)$ & $5(55,5)$ & $2(66,6)$ \\
\hline Naproxemo & 0 & $4(21,0)$ & $24(34,8)$ & $4(28,6)$ & $3(33,3)$ & $1(33,3)$ \\
\hline Ibuprofeno & 0 & 0 & $1(1,4)$ & 0 & 0 & 0 \\
\hline Diclofenaco+ & & & & & & \\
\hline Paracetamol & $1(16,7)$ & 0 & $1(1,4)$ & 0 & 0 & 0 \\
\hline Rofecoxib & 0 & $1(5,3)$ & 0 & $1(7,1)$ & $1(11,1)$ & 0 \\
\hline Total & 6 & 19 & 69 & 14 & 9 & 3 \\
\hline
\end{tabular}

FL: Frenectomía lingual; ER: Exodoncia de diente retenido; ES: Exodoncia de diente supernumerario; OD: Odontoma; OP: Operculectomía; EM: Enucleación de mucocele.

vero, luego de una cirugía oral, comparado con la administración de Paracetamol o Placebo.

Primosch et al. (15) encuentran que la administración pre-operatoria del Ibuprofeno, Acetaminofen o Placebo, muestran un 52\% de analgesia posexodoncia en niños de $2 \mathrm{a}$ 10 años de edad; no existiendo diferencias significativas en el uso de una u otra sustancia en el alivio del dolor referido.

Buljcik et al. (16) atribuyen una mayor analgesia posoperatoria al Ibuprofeno comparado con el Paracetamol, siendo mínimos los efectos adversos presentados luego del uso del Ibuprofeno administrado primero vía oral y luego rectal para el alivio del dolor después de tonsilectomias en niños.

Bennie et al. (17) encuentran que el uso del Ibuprofeno o Paracetamol como analgesia pre-operatoria oral en niños sometidos a miringotomìa mostró las mínimas ventajas que el uso del placebo en el manejo del dolor posoperatorio.

Pickering et al. (18) comparan la adición del Ibuprofeno con el Paracetamol y del Rofecoxib con el Paracetamol en la analgesia preoperatoria; concluyendo que el Ibuprofeno con el Paracetamol reduce la necesidad de analgésicos posoperatorio en un 38-72\% de los niños de 3 a 15 años sometidos a tonsilectomias.

Otros AINES utilizados en el estudio en menor proporción fueron: Diclofenaco, Rofecoxib, Naproxeno sódico. No obstante en la literatura no se encontraron estudios referentes a su aplicación en cirugías menores en odontopediatría.

Si bien es cierto, el Diclofenaco como tal no es recomendado en niños menores de 16 años; no obstante en nuestro estudio se utilizo Diclofenaco asociado al Paracetamol (Dolocordralan) para potencializar su efecto analgésico y disminuir sus efectos colaterales; teniendo en cuenta que la interacción farmacológica favorece el sinergismo de sus acciones.

En cuanto al Rofecoxib (Vioxx) su prescripción fue dada muy pocas veces y en periodos cortos; estuvo indicada en pacientes con susceptibilidad gástrica.

Finalmente la medicación analgésica en el paciente pediátrico va a depender del grado de respuesta (inflamación) que se obtenga de la intervención quirúrgica, dependiendo además de la invasión de tejidos, lo cual explicaría porque algunos medicamentos son indicados en el pre-operatorio, unos indicados en el posoperatorio, y otros en ambos casos. Siendo lo más importante en la decisión a tomar conseguir mayor confort del paciente pediátrico. 


\section{Conclusiones}

El tipo de cirugía oral menor mas frecuentemente practicado en pacientes atendidos en pacientes en el Servicio de Odontología Pediátrica fue la exodoncia de dientes supernumerarios en ambos sexos; mientras que según edad la exodoncia de dientes supernumerarios fue mayormente realizada en niños de 7 a 11 años a más; en el grupo de 4 a 6 a fue la frenectomía lingual y en el grupo de 1 a 3 a fueron la frenectomía lingual, enucleación de odontoma y eliminación de mucocele.

El tipo de analgésicos posquirúrgicos recetados en pacientes en el Servicio de Odontología Pediátrica según sexo, se encontró que el Ketorolaco fue el más recetado en el sexo masculino y el Paracetamol en el sexo femenino; según edad, el ketorolaco fue el más recetado en el grupo de 7 a11 y el Paracetamol en los demás grupos. Según tipo de cirugía el paracetamol fue más recetado en frenectomía lingual, operculectomía y eliminación de mucocele, y el ketorolaco en exodoncia de dientes retenidos, supernumerarios y enucleación de odontoma.

El número promedio de analgésicos posquirúrgicos en pacientes atendidos en el Servicio de Odontología Pediátrica fue valores mínimos y máximos de uno y cuatro medicamentos respectivamente. El número de analgésicos posquirúrgicos recetados fue mayor en el sexo masculino, en los niños de 7 a 11 años de edad y en la cirugía de exodoncia de dientes supernumerarios.

\section{Referencias bibliográficas}

1. Bejarano PF, Schmidt RF. Entendiendo el problema del dolor: mecanismos y semiología. Trib Med. 1993; 79(4):164-172.
2. Ziser A, Murray MJ. Postoperative pain. Analgesics make a difference in many ways. Postgrad Med. 1993; 93(2):1734, 177-80, 183-4.

3. Caceda J. Control del dolor, analgesia y sedación consciente en niños [monografía]. [Lima]: Univerisdad Peruana Cayetano Heredia; 1996.

4. Servicio de Medicina Pro Vida. Manual de Terapéutica Medica. Lima: Midzuaray A; 1998.

5. Pinkham J. R. Odontologia Pediatrica. México D.F.: Interamericana Mc Graw-Hill; 1991.

6. Katzung, Bertram G. Basic and Clinical Pharmacology. Mexico D.F.: El Manual Moderno; 1994.

7. Goodman Gilman Alfred, Joel G, Hardman. Las Bases Farmacológicas de la Terapéutica. 1. 10ma ed. México: Interamericana Mc Graw Hill; 2003.

8. Pérez A, Lopez A, Grau I. Antiinflamatorios no esteroideos (AINES): Consideraciones para su uso estomatológico. Rev Cubana Estomatol. 2002; 39(2):119138.

9. Barraza O. Manejo del dolor post-operatorio en cirugía pediátrica: Encuesta comparativa siete y catorce anos después. Diagnostico. 2005; 44(2):60-6.

10. Barraza O. Encuesta a 50 cirujanos pediátricos de Lima sobre el uso de antálgicos en niños postoperados. Diagnóstico. 1995; 34(4):25-31.

11. Jürgens $S$, Warwick RS, Inglehearn PJ, Gooneratne DS. Pain relief for paediatric dental chair anaesthesia: current practice in a community dental clinic. Int J Paediatr Dent. 2003; 13(2):93-7.

12.Pedreira MF, Vadillo JM, Maqueda MJ, Manso FJ, García
Nuñez JA. Estudio controlado sobre la eficacia y tolerabilidad de ibuprofeno frente a paracetamol en el tratamiento del dolor postextracción dental en pacientes pediátricos. Av Odontoestomatol. 1999; 15(4):209-14.

13. Haas DA. An update on analgesics for the management of acute postoperative dental pain. J Can Dent Assoc. 2002; 68(8):476-82.

14. Mehlisch DR, Sollecito WA, Helfrick JF, Leibold DG, Markowitz R, Schow CE Jr, Shultz R, Waite DE. Multicenter clinical trial of ibuprofen and acetaminophen in the treatment of postoperative dental pain. J Am Dent Assoc. 1990; 121(2):257-63.

15.Primosch RE, Nichols DL, Courts FJ. Comparison of preoperative ibuprofen, acetaminophen, and placebo administration on the parental report of postextraction pain in children. Pediatr Dent. 1995; 17(3):187-91.

16.Buljcik M, Milosevic D, Komazec Z. [Relief of pain after tonsillo-adenoidectomy in preschool-age children]. Srp Arh Celok Lek. 2002; 130(Suppl 1):40-3.

17.Bennie RE, Boehringer LA, McMahon S, Allen H, Dierdorf SF. Postoperative analgesia with preoperative oral ibuprofen or acetaminophen in children undergoing myringotomy. Paediatr Anaesth. 1997; 7(5):399-403.

18. Pickering AE, Bridge HS, Nolan J, Stoddart PA. Double-blind, placebo-controlled analgesic study of ibuprofen or rofecoxib in combination with paracetamol for tonsillectomy in children. $\mathrm{Br}$ J Anaesth. 2002; 88(1):72-7. 